\section{CVIA}

REVIEW ARTICLE

pISSN 2508-707X / eISSN 2508-7088 https://doi.org/10.22468/cvia.2017.00157 CVIA 2018;2(1):19-27

\title{
Stress-Induced Cardiomyopathy: Assessment with Cardiac Magnetic Resonance Imaging and Multi-Detector Computed Tomography
}

\author{
Yeonah Kang 1,2, Yeo Koon Kim², Eun Ju Chun², Sang II Choi \\ 'Department of Radiology, SMG-SNU Boramae Medical Center, Seoul, Korea \\ 2Department of Radiology, Seoul National University Bundang Hospital, Seongnam, Korea
}

Received: September 28, 2017

Revised: December 11, 2017

Accepted: December 11, 2017

Corresponding author

Sang \| Choi, MD

Department of Radiology,

Seoul National University

Bundang Hospital,

82 Gumi-ro 173beon-gil,

Bundang-gu, Seongnam 13620, Korea

Tel: 82-31-787-7609

Fax: 82-31-787-4011

E-mail: drsic@hanmail.net
Stress-induced cardiomyopathy is an increasingly recognized disease entity that has been recently accepted as a unique form of reversible cardiomyopathy. Cardiac magnetic resonance imaging (MRI) and multi-detector computed tomography (MDCT) are useful modalities for evaluating regional wall motion abnormalities, patterns of left ventricular (LV) ballooning, and coronary artery stenosis. Thus, cardiac MRI and MDCT may be useful for distinguishing stressinduced cardiomyopathy from acute coronary syndrome. This pictorial essay provides an overview of the imaging findings of stress-induced cardiomyopathy, including the different patterns of LV ballooning. In addition, the potential roles of cardiac MRI and MDCT in evaluating stress-induced cardiomyopathy are discussed.

Key words Stress-induced cardiomyopathy · Magnetic resonance imaging Multidetector computed tomography.

\section{INTRODUCTION}

Stress-induced cardiomyopathy is a unique form of cardiomyopathy characterized by reversible left ventricular (LV) wall dyskinesis-akinesis with absence of significant epicardial coronary lesions [1-4]. Stress-induced cardiomyopathy is also known as Takotsubo cardiomyopathy after the Japanese name for "octopus pot," and was given this name due to the characteristic balloon shape of the LV apex seen in the disease [5]. Although stress-induced cardiomyopathy is more prevalent among men in Japan [6,7], it is greater among women in western countries [5,7-9].

Stress-induced cardiomyopathy is generally recognized as a benign disorder [5]; however, the clinical features of the disease fall along a diverse spectrum [7]. Most patients with stress-induced cardiomyopathy present with acute chest pain or dyspnea with associated electrocardiographic changes and/or elevated

(a) This is an Open Access article distributed under the terms of the Creative Commons Attribution Non-Commercial License (http://creativecommons.org/licenses/bync/4.0) which permits unrestricted non-commercial use, distribution, and reproduction in any medium, provided the original work is properly cited. cardiac enzyme levels $[3,8,10]$. Thus, as patients with stress-induced cardiomyopathy commonly present with symptoms similar to those of acute coronary syndrome, the initial diagnosis and treatment of patients in the emergency room remains challenging [11]. The purpose of this article is to provide an overview of the potential pathophysiology of stress-induced cardiomyopathy and to describe the clinical workflow, imaging findings, different patterns of LV ballooning, and potential roles of cardiac magnetic resonance imaging (MRI) and multidetector computed tomography (MDCT) in the evaluation of this disease process.

\section{POTENTIAL PATHOPHYSIOLOGY AND CLINICAL WORKFLOW OF STRESS- INDUCED CARDIOMYOPATHY}

Stress-induced cardiomyopathy is a well-known but enigmatic disease that is often triggered by physical as well as emotional stress, but can also occur in the absence of any triggers [5,7]. Therefore, the exact pathophysiology of stress-induced cardio- 
myopathy remains controversial, although it seems that many factors contribute to the disease, including microvascular, endocrine, and neurologic etiologies [3,7,12-14].

The Mayo Clinic has proposed diagnostic criteria for stressinduced cardiomyopathy, which comprise 1) transient hypokinesis, akinesis, or dyskinesis of the LV mid segments with or without apical involvement, 2) absence of obstructive coronary disease or angiographic evidence of acute plaque rupture, 3 ) new electrocardiography (ECG) abnormalities (either ST-segment elevation and/or $\mathrm{T}$ wave inversion) or modest elevations in cardiac troponin levels, and 4) absence of pheochromocytoma or myocarditis [15]. However, troponin levels and ECG changes in the emergency department are not sufficient to differentiate stress-induced cardiomyopathy from acute coronary syndrome [7], and thus early coronary angiography remains necessary to rule out the latter. Notably, $15.3 \%$ of patients with stress-induced cardiomyopathy have evidence of coexisting coronary artery disease on angiography, suggesting that the presence of coronary artery disease is not an appropriate criterion for excluding a diagnosis of stress-induced cardiomyopathy $[15,16]$.

In order to demonstrate the characteristic morphological changes of LV ballooning in stress-induced cardiomyopathy, invasive left ventriculography has been used. Considering the fact that invasive coronary angiography is an established routine practice for early reperfusion therapy in the clinical setting of ST-elevation myocardial infarction (STEMI), and also that classic stress-induced cardiomyopathy mimics the clinical features of STEMI, an invasive diagnostic work-up is believed to be inevitable [17]. However, the diverse clinical situations associated with development of stress-induced cardiomyopathy have raised an important clinical issue regarding the need for a noninvasive method for diagnosing stress-induced cardiomyopathy. Indeed, it is often not feasible for critically ill patients in the intensive care unit or post-general anesthesia recovery unit to undergo invasive imaging studies $[6,18]$.

\section{POTENTIAL ROLE OF NON-INVASIVE MULTI-MODALITY IMAGING IN STRESS- INDUCED CARDIOMYOPATHY}

Along with cumulative clinical experience, cardiac imaging specialists have become increasingly familiar with the various patterns of LV ballooning, and noninvasive imaging modalities such as echocardiography or MRI may successfully replace invasive ventriculography [19-21].

\section{Echocardiography}

Over the past several years, echocardiography has been shown to play a pivotal role in the accurate evaluation of LV function in diverse cardiac disease as well as stress-induced cardiomyopathy [22]. Echocardiography has several advantages, including its ability to achieve easy and rapid bedside assessment, especially in critically ill patients requiring complex care in the intensive care unit or emergency department. In these patients, an invasive diagnostic work-up is not infrequently contraindicated or impossible, supporting the need for new diagnostic approaches [8]. However, there are some limitations of echocardiography that impede its utility in obtaining an accurate diagnosis. Specifically, echocardiography is an operator-dependent technique and is influenced by the acoustic window [8].

\section{Coronary angiography}

Coronary angiography is a confirmatory tool used to evaluate the absence of obstructive coronary artery disease and obtain evidence of acute plaque rupture. Most patients who undergo coronary angiography exhibit normal coronary arteries or have insignificant coronary artery stenosis [9]. Thus, a challenging issue with this technique is excluding the possibility of obstructive epicardial coronary artery disease and acute plaque rupture. In the past, clinicians have focused on the clinical implications of stress-induced cardiomyopathy as an important component of the differential diagnosis of acute coronary syndrome or STEMI, and invasive coronary angiography should indeed be routinely performed in acutely ill patients suspected of having one of these conditions. On the other hand, there have been several recent reports of stress-induced cardiomyopathy diagnosed based on echocardiographic demonstration of typical LV morphological change and clinical course without the need for invasive coronary angiography [23,24].

\section{Nuclear imaging}

Recent studies have reported a potential role of nuclear imaging findings in stress-induced cardiomyopathy. Single-photon emission computed tomography can assess neurogenic myocardial stunning, a consequence of defects in myocardial perfusion and sympathetic innervation, using ${ }^{201}$ Thallium or ${ }^{99 m}$ Technetium-labelled radiopharmaceuticals and ${ }^{123} \mathrm{I}$-metalodobenzylguanidine (I-123 MIBG). Likewise, 18F-fluorodeoxyglucose (FDG) positron emission tomography can be used to detect myocardial glucose metabolism $[25,26]$. In the acute and subacute phases of stress-induced cardiomyopathy, the affected hypocontractile ventricular segment shows defects of MIBG and FDG uptake despite normal to slightly reduced perfusion. Subsequent rapid normalization of myocardial perfusion and delayed recovery of both LV glucose metabolism and sympathetic innervation are also observed $[25,26]$.

\section{Cardiac multi-detector computed tomography}

MDCT is a comprehensive modality for evaluating both car- 
diac function and coronary artery lesions when combined with retrospective ECG gating (Fig. 1). Therefore, MDCT provides useful information regarding regional wall motion abnormalities, and can immediately rule out coronary artery lesions with a high negative predictive value $[27,28]$. In addition, cardiac MDCT can be used to promptly evaluate both cardiac and noncardiac causes of acute chest pain in the emergency department. One previous study reported that cardiac MDCT, conducted as part of a triple-rule-out CT angiography approach, can reduce the time for triage [29]. In clinical practice, excluding the possibility of obstructive epicardial coronary artery disease or acute plaque rupture with MDCT remains challenging. However, cardiac MDCT with prospectively ECG-gating can achieve significant dose reduction compared to retrospective ECG-gated techniques for ruling out obstructive epicardial coronary artery disease. Therefore, cardiac MDCT may be an accessible alternative for evaluating suspected stress-induced cardiomyopathy [8].

\section{Cardiac magnetic resonance imaging}

Cardiac MRI is a non-invasive unique modality that provides information regarding tissue characteristics, cardiac function, and structural abnormalities. Cardiac MRI is also helpful for distinguishing between causes of reversible injury such as inflammation and ischemic edema and causes of irreversible injury such as necrosis and fibrosis. Moreover, compared to echocardiography, cardiac MRI is less operator-dependent and is not subject to acoustic window limitations. Cine MRI with the steady-state free precession pulse sequence offers advantages of multiplanar imaging, complete coverage of the entire myocardium without obliquity, and excellent soft-tissue contrast between the myocardial border and blood pool. Thus, cardiac MRI can allow for better characterization of the pattern and distribution of LV ballooning in patients with stress-induced cardiomyopathy $[2,4,30,31]$. Further, many studies have reported that late gadolinium enhancement (LGE) is generally absent in stress-induced cardiomyopathy compared to acute myocardial infarction $[2,32,33]$.

Diagnostic criteria for stress-induced cardiomyopathy based on cardiac MRI have been proposed by several studies, and comprise 1) severe LV dysfunction in a non-coronary regional distribution pattern on cine MRI, 2) myocardial edema on T2 weighted images indicative of acute ischemia or inflammation, colocalized with regional wall motion abnormalities, 3) and absence of high-signal areas on LGE images [30]. Therefore, the entire MR imaging protocol for stress-induced cardiomyopathy should include cine MRI, T2 weighted, and LGE sequences.

Several recent studies have reported that subtle myocardial enhancement may be present in patients with stress-induced cardiomyopathy on delayed enhancement MRI $[11,30,34]$. The critical difference with the enhancement observed in myocardial infarction and myocarditis is the cutoff value used to determine its presence. With respect to cut-off values, there is no enhancement in cases of stress-induced cardiomyopathy based on a signal intensity threshold of 5-standard deviations, whereas myocardial infarction and myocarditis exhibit enhancement at that level [34].

The time interval between symptom onset and cardiac MRI scan can also influence myocardial enhancement. A prospective study showed that hearts with stress-induced cardiomyopathy exhibit mild transmural enhancement in the affected area on cardiac MRI when performed within 72 hours of the onset of symptoms, which may correspond to localized inflammation and edema [11]. The same study reported that follow-up MRI
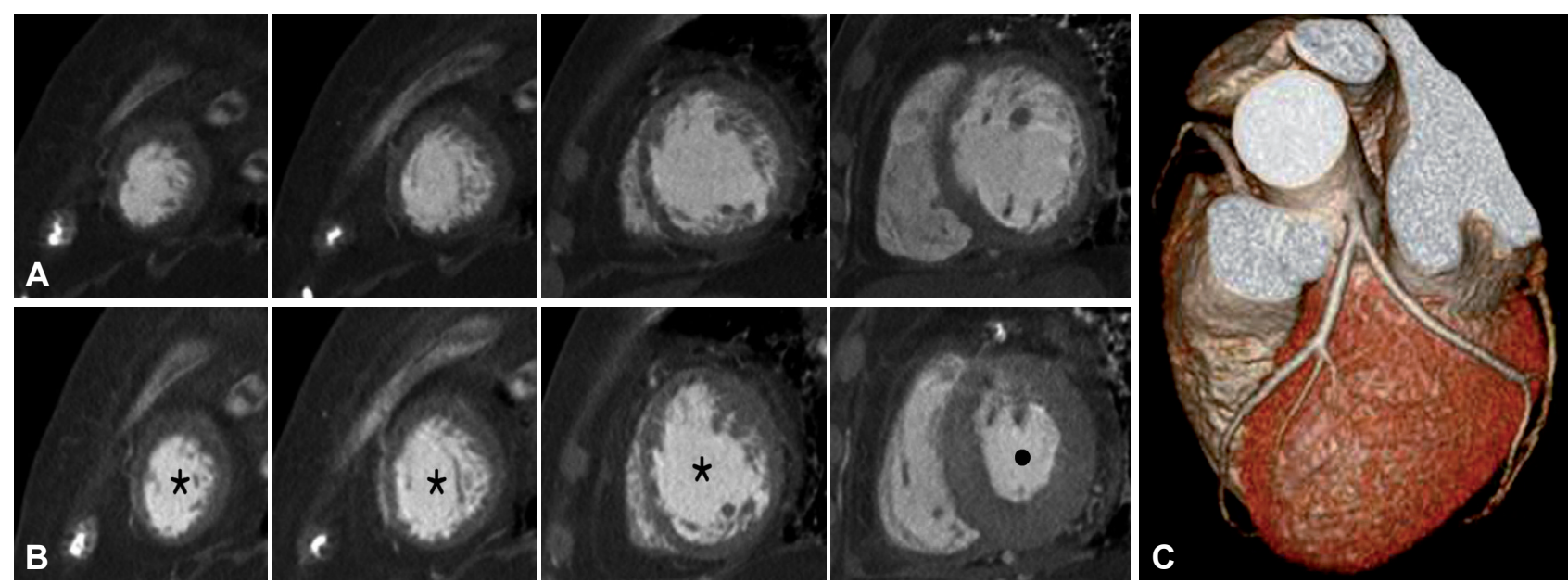

Fig. 1. A 70-year-old female with acute chest pain and elevated cardiac enzymes. (A) End diastolic short axis view and (B) end systolic short axis view on cardiac multi-detector computed tomography. Global hypokinesia (asterisk) is noted at the mid to apical LV wall with a hypercontractile myocardium (circle) of the LV base. However, a normal coronary artery is seen on a (C) three-dimensional volume rendering image. LV: left ventricular. 
performed after 90 days of the onset of symptoms generally shows resorption of edema, improved contractility, and return of the late-enhancement image findings [11]. A study by Rolf et al. [35] utilized an immunohistochemistry approach to identify transient fibrosis as a potential cause of LGE in stress-induced cardiomyopathy, whereas necrosis or replacement of fibrosis was found to be the cause of LGE in myocardial infarction.

Lastly, cardiac MRI may have a potential role in predicting the prognosis of patients with stress-induced cardiomyopathy patients. Specifically, previous clinical research aimed at evaluating the relationship between LGE and prognosis in stress-in-

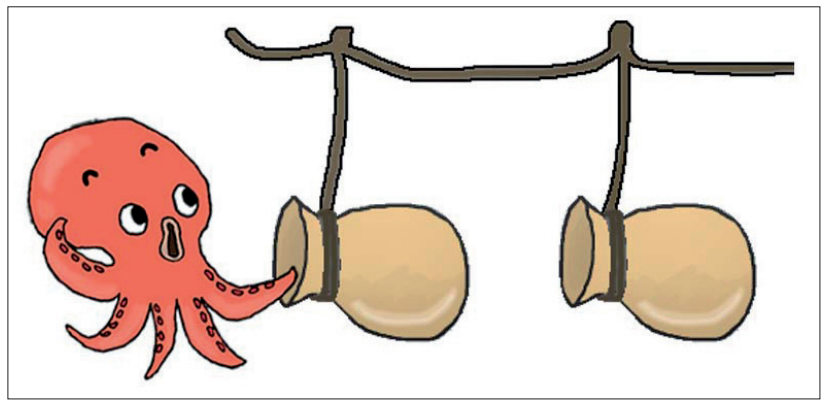

Fig. 2. A Takotsubo, the Japanese name for octopus trap, which has a narrow neck with a round base. duced cardiomyopathy showed that patients with LGE are more likely to exhibit cardiogenic shock and have a longer duration for electrocardiography normalization and recovery of wall motion compared to patients without LGE [36].

\section{MORPHOLOGIC SPECTRUM OF STRESS- INDUCED CARDIOMYOPATHY}

Stress-induced cardiomyopathy is also known as Takotsubo cardiomyopathy after the Japanese word for octopus trap (Fig. 2), which is similar in shape to the apical ballooning of the left ventricle seen during systole in the typical form of this disorder [3]. Along with cumulative clinical experience, different patterns of LV ballooning have been observed, and stress-induced cardiomyopathy is classified according to one of four patterns of LV ballooning [7] (Fig. 3). A recent study reported that among 1750 patients with stress-induced cardiomyopathy, the most common pattern is apical type ( $81.7 \%$ of patients), followed by mid-ventricular type (14.6\%), basal type (2.2\%), and focal type (1.5\%) [7]. Thus, the morphologic spectrum of stress-induced cardiomyopathy is broad, ranging from well-known apical ballooning to atypical wall motion abnormalities. Importantly, the potential differences in clinical features and outcomes according
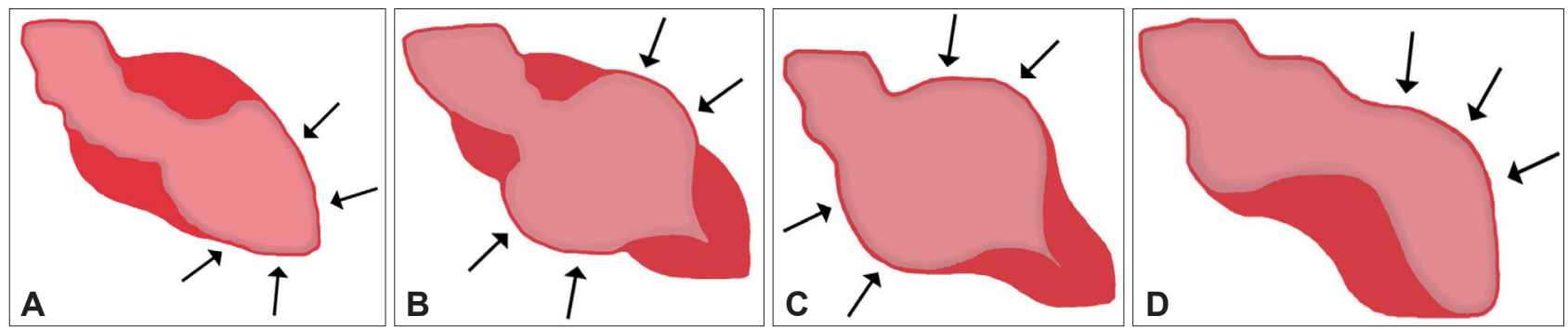

Fig. 3. Schematic diagram of the different patterns of LV ballooning seen at the end systolic phase. (A) LV apical ballooning. (B) Mid-ventricular ballooning. (C) Basal ballooning. (D) Focal type. LV: left ventricular.
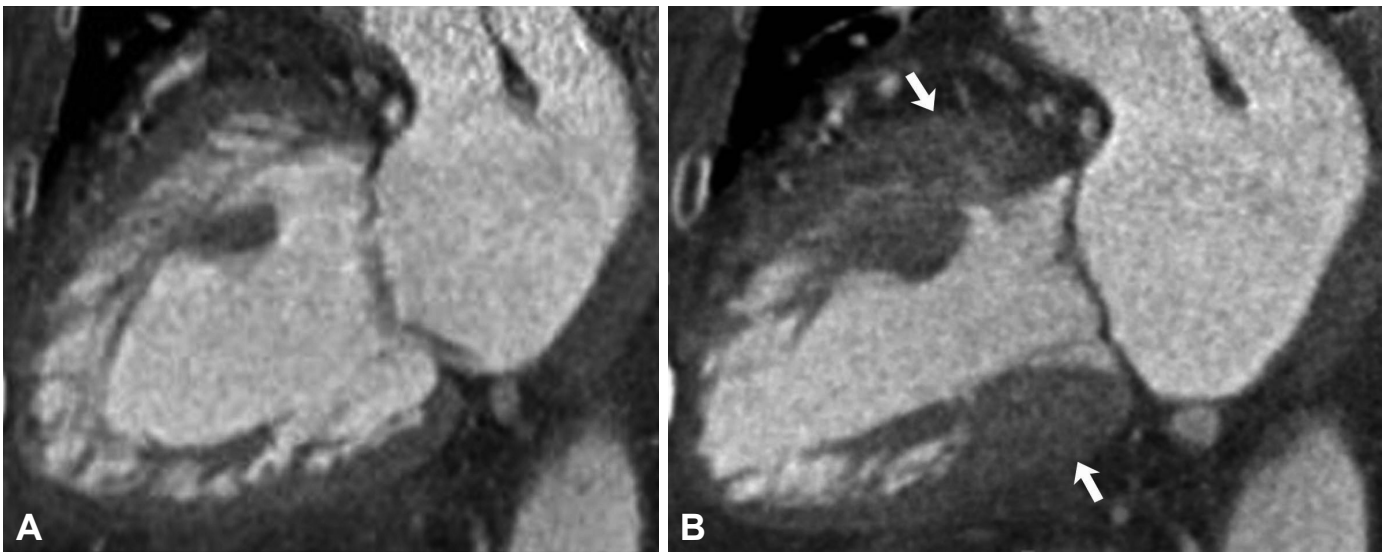

Fig. 4. Underlying non-compaction of LV with apical ballooning type. End diastolic 2 chamber view (A) and end systolic 2 chamber view (B) of multi-detector computed tomography images showing typical apical ballooning type of stress-induced cardiomyopathy. Note the underlying non-compaction of LV on end diastolic imaging and global hypokinesia except the basal wall on end systolic images (arrows). LV: left ventricular. 
to these different patterns of wall motion abnormalities need to be evaluated further [8].

\section{Apical ballooning (typical stress-induced cardiomyopathy)}

LV apical ballooning is associated with the so-called typical stress-induced cardiomyopathy [3], and presents with basal hyperkinesia and apical ballooning (Figs. 4 and 5).

\section{Mid-ventricular ballooning}

Mid-ventricular ballooning type presents as an isolated akinesia of mid-ventricular level. Hyper-contractile motion of the LV apex and base with ballooning of the mid-ventricle segments is seen in this form of stress-induced cardiomyopathy (Fig. 6) [37].

\section{Basal ballooning}

Basal ballooning type is often called reverse or inverted stressinduced cardiomyopathy, as it shows apical hyperkinesia with basal sparing (Fig. 7) [38].

\section{Focal type}

Focal type does not have fixed morphological pattern $[7,39]$, and can present as either focal wall motion abnormalities or dyssynchrony of the left ventricle (Fig. 8, Supplementary Movie 1 in the online-only Data Supplement).
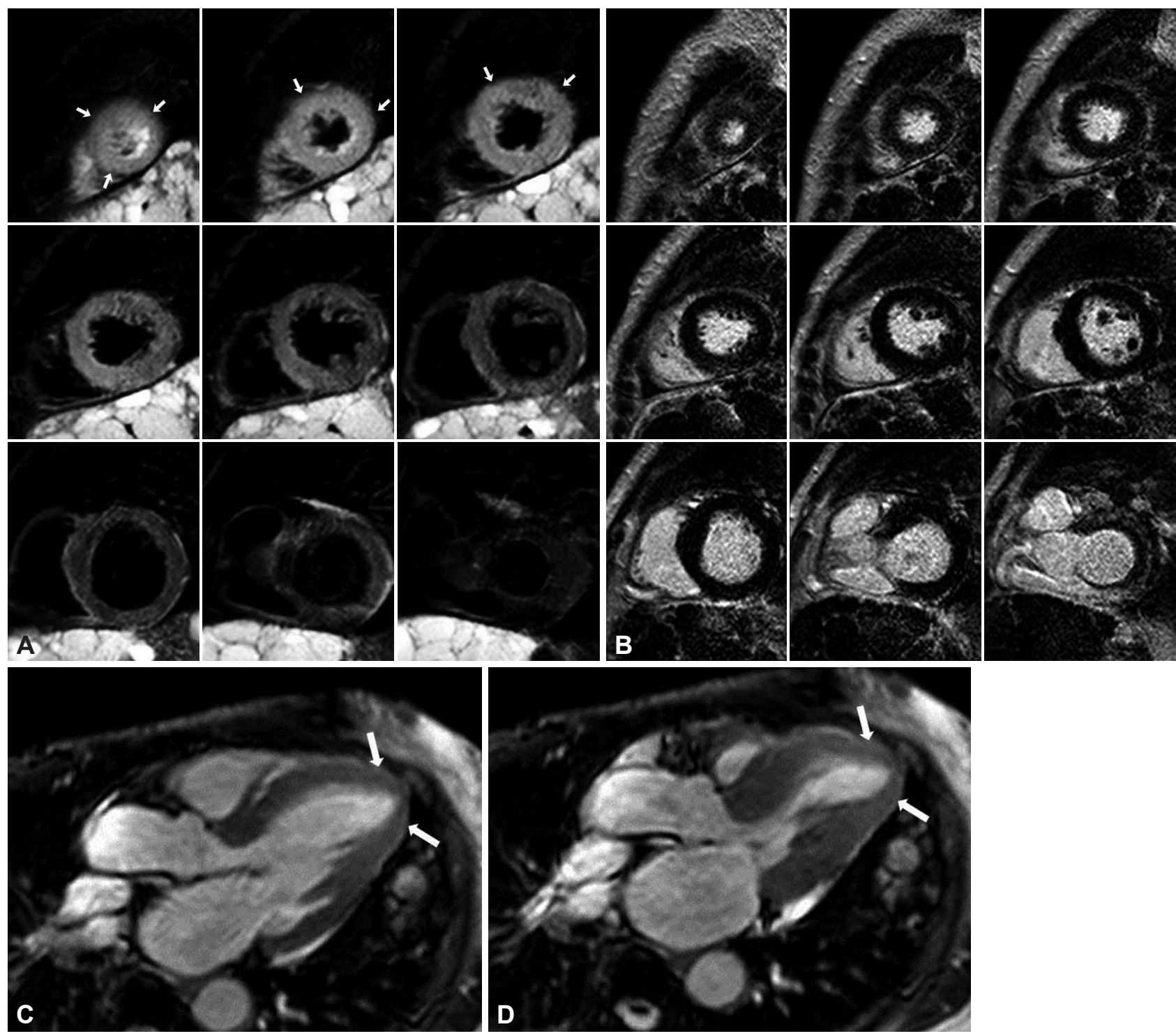

Fig. 5. Cardiac magnetic resonance imaging of the apical ballooning type. (A) T2 signal increase in apical and lower portion of mid-ventricular LV wall (arrows). (B) There is no definite abnormal late enhancement within the myocardium of the LV. End diastolic 3 chamber view (C) and end systolic 3 chamber view (D) of the cine image. The cine image shows hyperkinesia of basal segment and apical ballooning (arrows). 


\section{DIFFERENTIAL DIAGNOSIS}

Differentiating apical ballooning type stress-induced cardiomyopathy from apical-anterior acute myocardial infarction remains a clinical challenge. A previous study found that the clinical, laboratory, and echocardiography findings of apical ballooning type stress-induced cardiomyopathy are not significantly different from the findings associated with acute myocardial infarction due to mid-distal left anterior descending artery occlusion [40]. In this particular situation, echocardiography is unable to reliably diagnose stress-induced cardiomyopathy, because $80 \%$ of acute myocardial infarction patients exhibit hypoakinesia of all LV apical segments [40].

The critical differential diagnosis of stress-induced cardiomyopathy is acute coronary syndrome, especially acute myocardial infarction. On imaging, typical acute myocardial infarc-
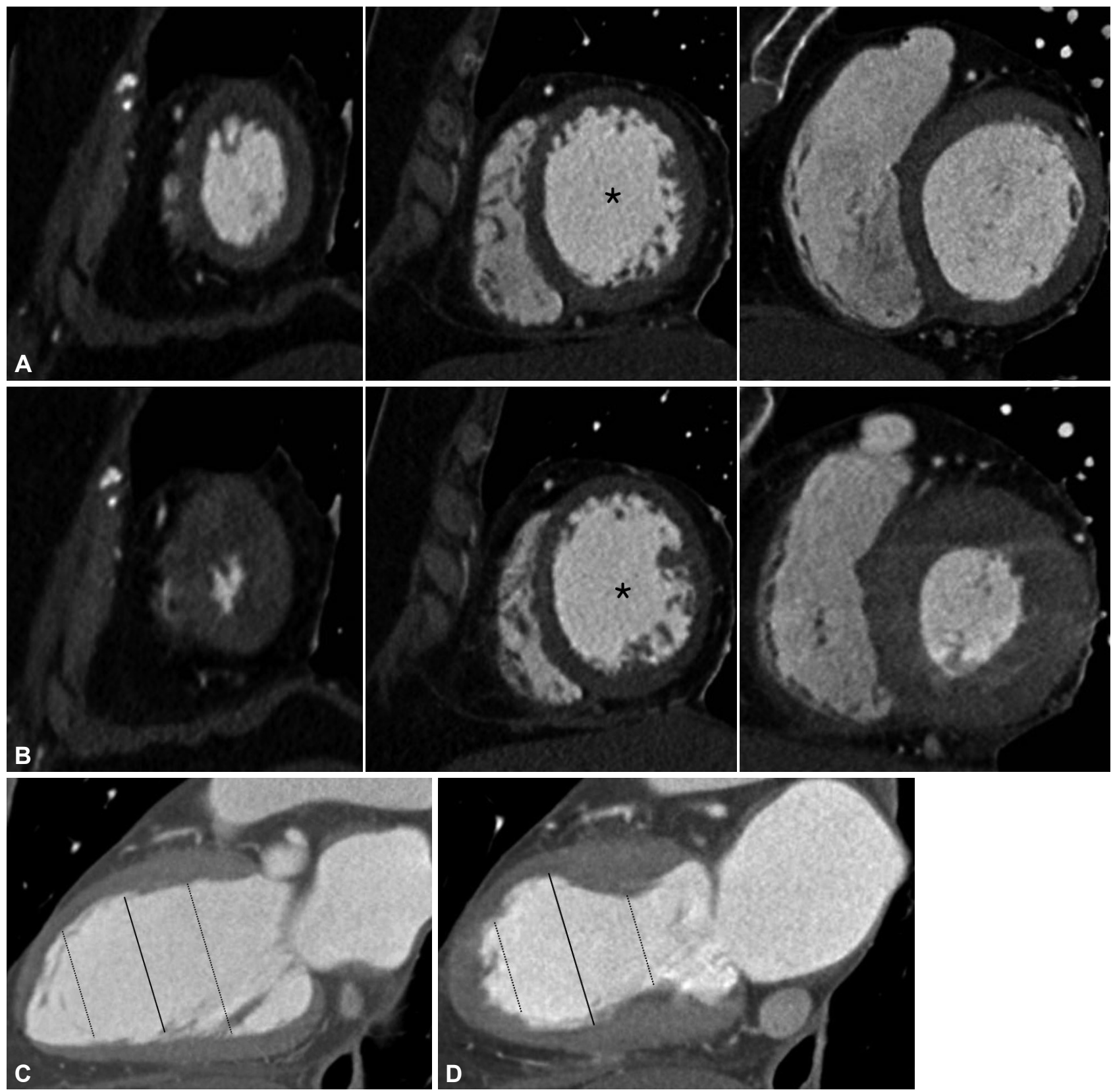

Fig. 6. Mid-ventricular ballooning type. (A) End diastolic phase, short axis view. (B) End systolic phase, short axis view. (C) End diastolic phase, 2 chamber view. (D) End systolic phase, 2 chamber view. A 63-year-old female with acute chest discomfort underwent cardiac multidetector computed tomography, which showed akinesia vs severe hypokinesia of the entire LV wall of the mid-ventricle (A, B, C, and D: asterisk, solid line) and a relatively hyperkinetic basal and apical wall (C and D: dot line). This patient was finally diagnosed with mid-ventricular type stress-induced cardiomyopathy. Quantitative analysis of cardiac function data revealed mild LV dysfunction $(E F=46.3 \%$, LVEDV $=123.49$ $\mathrm{mL}$, LVESV=66.37 mL). LV: left ventricular. 
tion shows significant epicardial coronary artery stenosis or occlusion [41], and cardiac MRI can be used for the diagnosis of myocardial infarction. With cardiac MRI, the LGE sequence detects nonviable myocardium with strong enhancement along the vascular territory in patients with myocardial infarction, while T2-weighted MRI has the potential to differentiate edema and fibrosis, which are suggestive of acute and chronic injury, respectively $[23,42]$. These finding present along the coronary artery distribution with wall motion abnormalities at the corresponding area (Fig. 9).

In addition to acute coronary syndrome, myocardial inflam- mation caused by viral or post-viral autoimmune responses, specific pathogens such as bacteria, drugs, and chemicals is part of the differential diagnosis of stress-induced cardiomyopathy. Most patients present with flu-like symptoms and frequently have elevated cardiac enzymes [43]. With the LGE sequence of cardiac MRI, acute myocarditis appears as multifocal patchy enhancement at the non-vascular territory. In addition, edema can present predominantly at the epicardial region on T2 weighted images (Fig. 10) [43,44].
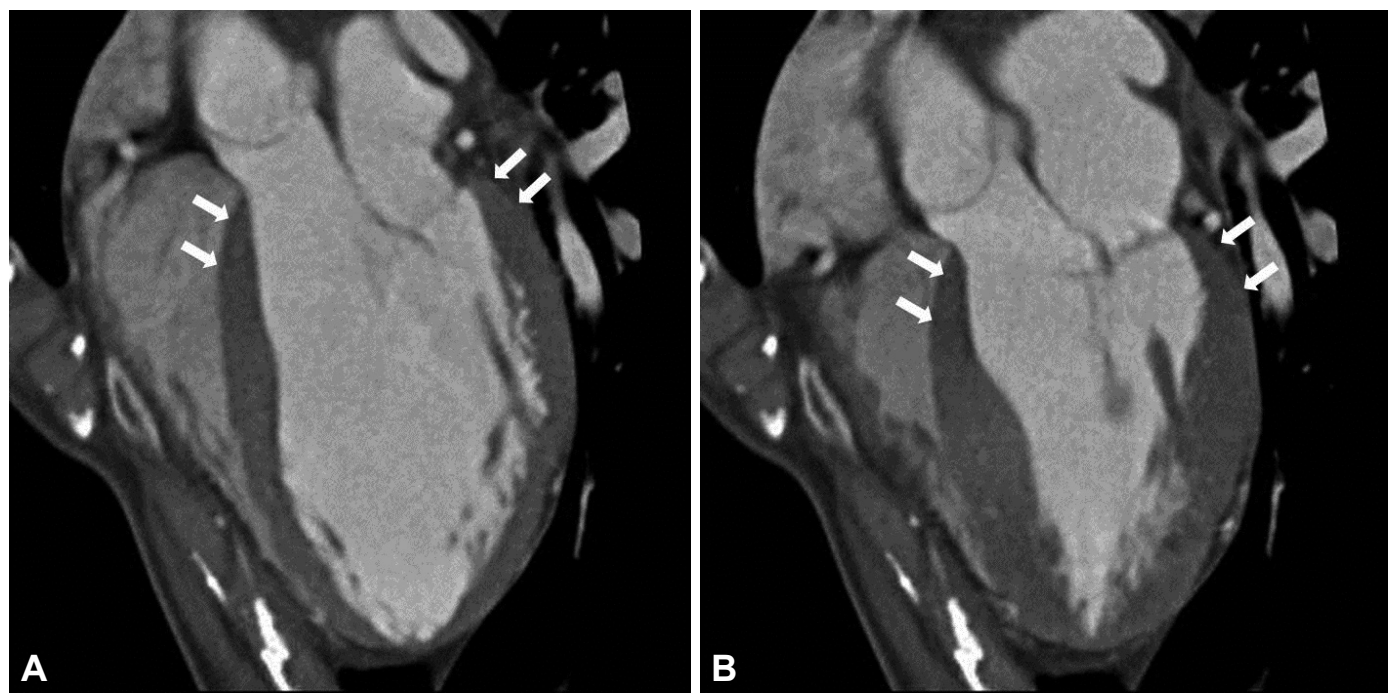

Fig. 7. Basal ballooning type. A 55-year-old female was confirmed as having a basal ballooning pattern of stress-induced cardiomyopathy. (A) End diastolic phase, 3 chamber view. (B) End systolic phase, 3 chamber view. In this case, cardiac CT demonstrated hypokinesia of the entire basal wall (arrows) and hyperkinesia of the entire apical to mid left ventricular wall.
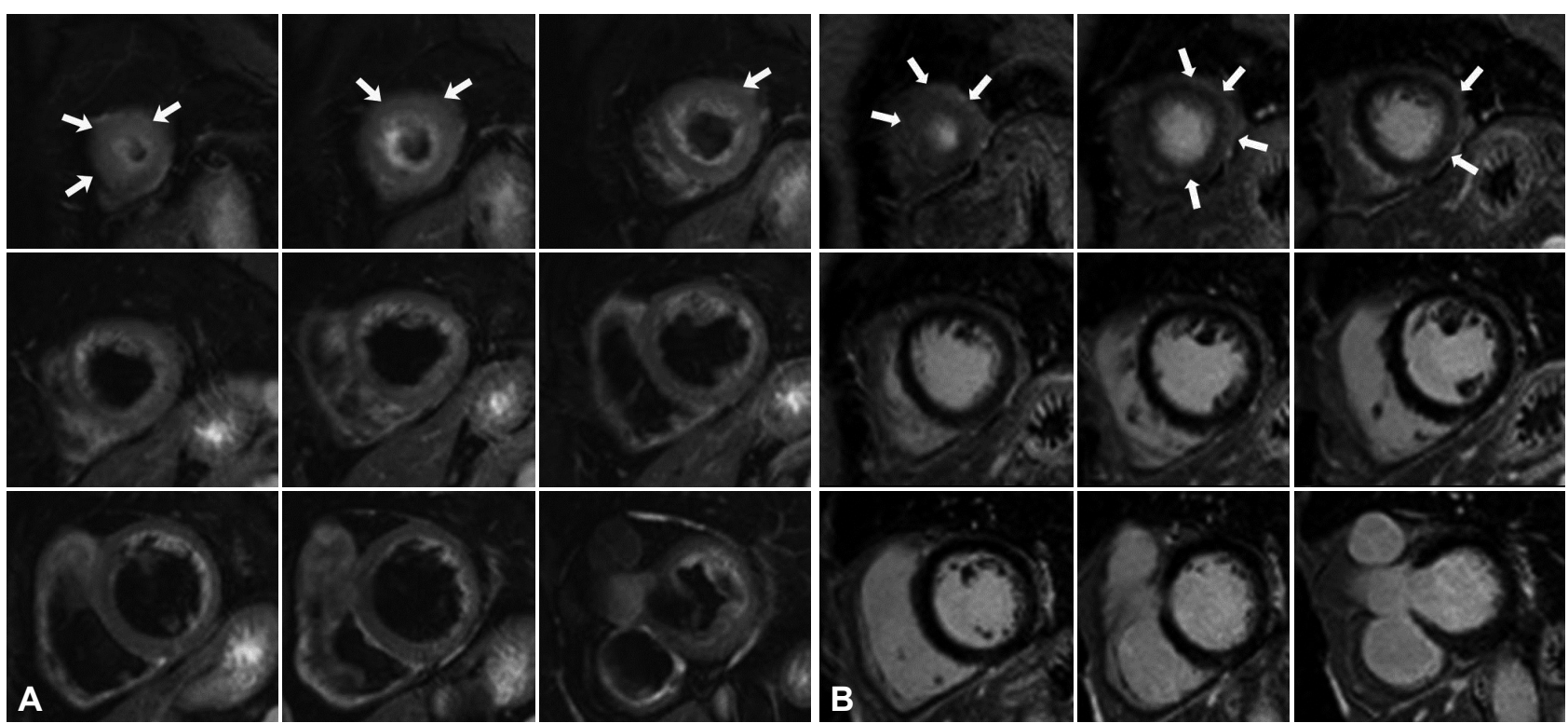

Fig. 8. A 64-year-old female was diagnosed with focal type stress-induced cardiomyopathy. She underwent cardiac magnetic resonance imaging within 24 hours of the onset of symptoms. (A) T2-weighted image showing a slightly high signal intensity of the left ventricular apex and entire apical wall (arrows) with mild fussy late enhancement (arrows) at the corresponding area on (B) a late gadolinium enhancement image. 

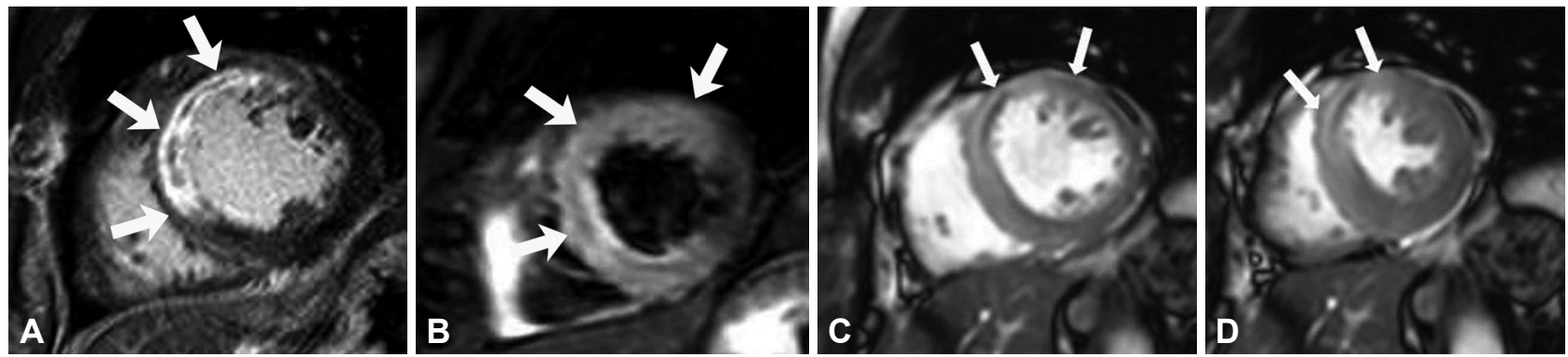

Fig. 9. A 45-year-old male with severe chest pain was diagnosed with acute myocardial infarction. Coronary angiography revealed total occlusion of the proximal LAD. (A) Cardiac DE-MRI showing delayed enhancement (transmural extent: 75-100\%) at the midventricular anterior and anteroseptal wall (arrows). (B) T2-weighted image showing diffuse high signal intensity at the corresponding area (arrows). (C) End diastolic phase, short axis cine view and (D) end systolic phase, short axis cine view show akinesia at the affected area (arrows) comprising the mid-ventricular anterior and anteroseptal wall along the vascular territory.
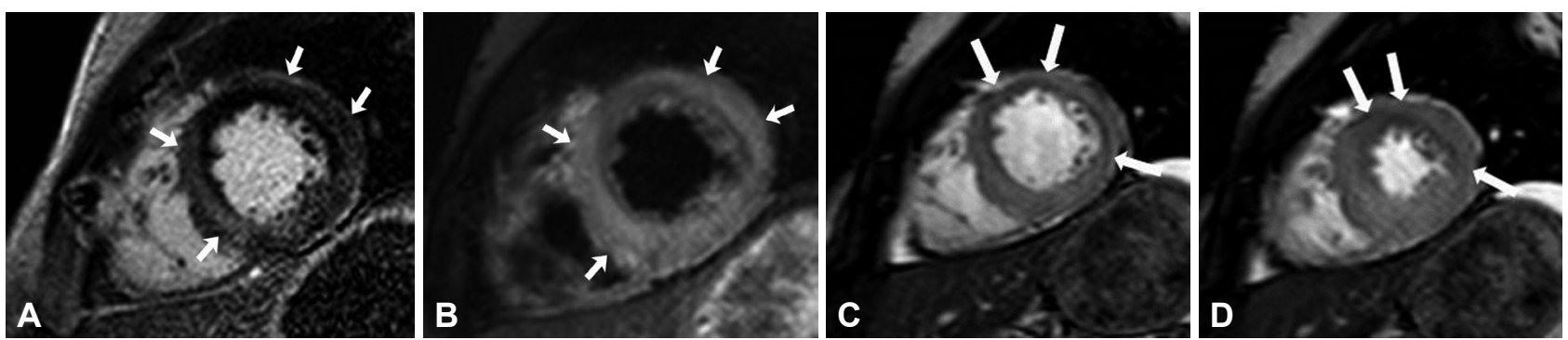

Fig. 10. A 28-year-old male with acute chest pain, fever, and increased cardiac enzyme levels underwent cardiac MRI. He was finally diagnosed with acute myocarditis. Cardiac MRI showing multifocal, patchy enhancements with non-vascular territory at mid to epicardial layer of midventricular anteroseptal, anterolateral and inferolateral wall (A), and a high signal intensity of the corresponding area was present in a (B) T2-wieghted image. The patient was finally diagnosed with acute myocarditis. (C) End diastolic phase, short axis cine view. (D) End systolic phase, short axis cine view. Hypokinesia was noted at the anterior and inferolateral wall (arrows) suggestive of regional wall motion abnormalities in a non-coronary pattern. MRI: magnetic resonance imaging.

\section{CONCLUSIONS}

Cardiac MRI and MDCT are unique tools for evaluating and characterizing patients suspected of having stress-induced cardiomyopathy. Likewise, cardiac MDCT can quickly provide both an assessment of coronary lesions and LV wall motion in patients with acute chest pain. Cardiac MRI can also be used to accurately visualize regional wall motion abnormalities, and allows for characterization of tissue that in turn can be used to differentiate stress-induced cardiomyopathy from acute coronary syndrome. Lastly, cardiac MRI has a potential role in predicting the prognosis of patients with stress-induced cardiomyopathy.

\section{Supplementary Movie Legend}

Movie 1. Four chamber view of cine MRI showing regional wall motion abnormality with dyssynchrony at the apex, apical septal, and apical to mid lateral wall.

\section{Supplementary Materials}

The online-only Data Supplement is available with this article at https:// doi.org/10.22468/cvia.2017.00157.

\section{Conflicts of Interest}

The authors declare that they have no conflict of interest.

\section{Acknowledgments}

This work was supported by a National Research Foundation of Korea (NRF) grant funded by the Korea government (MEST) (No. 2011-0023624).

\section{REFERENCES}

1. Sharkey SW, Lesser JR, Maron BJ. Cardiology patient page. Takotsubo (stress) cardiomyopathy. Circulation 2011;124:e460-e462.

2. Kono T, Sabbah HN. Takotsubo cardiomyopathy. Heart Fail Rev 2014; 19:585-593.

3. Akashi YJ, Goldstein DS, Barbaro G, Ueyama T. Takotsubo cardiomyopathy: a new form of acute, reversible heart failure. Circulation 2008;118: 2754-2762.

4. Bossone E, Lyon A, Citro R, Athanasiadis A, Meimoun P, Parodi G, et al. Takotsubo cardiomyopathy: an integrated multi-imaging approach. Eur Heart J Cardiovasc Imaging 2014;15:366-377.

5. Bybee KA, Kara T, Prasad A, Lerman A, Barsness GW, Wright RS, et al. Systematic review: transient left ventricular apical ballooning: a syndrome that mimics ST-segment elevation myocardial infarction. Ann Intern Med 2004;141:858-865.

6. Park JH, Kang SJ, Song JK, Kim HK, Lim CM, Kang DH, et al. Left ventricular apical ballooning due to severe physical stress in patients admitted to the medical ICU. Chest 2005;128:296-302.

7. Templin C, Ghadri JR, Diekmann J, Napp LC, Bataiosu DR, Jaguszewski $\mathrm{M}$, et al. Clinical features and outcomes of Takotsubo (stress) cardiomyopathy. N Engl J Med 2015;373:929-938.

8. Song J.K. Stress-induced cardiomyopathy. Curr Cardiovasc Imaging Rep 2011;4:406-415.

9. Parodi G, Del Pace S, Carrabba N, Salvadori C, Memisha G, Simonetti I, et al. Incidence, clinical findings, and outcome of women with left ven- 
tricular apical ballooning syndrome. Am J Cardiol 2007;99:182-185.

10. Bybee KA, Prasad A. Stress-related cardiomyopathy syndromes. Circulation 2008;118:397-409.

11. Avegliano G, Huguet M, Costabel JP, Ronderos R, Bijnens B, Kuschnir P, et al. Morphologic pattern of late gadolinium enhancement in Takotsubo cardiomyopathy detected by early cardiovascular magnetic resonance. Clin Cardiol 2011;34:178-182.

12. Paur H, Wright PT, Sikkel MB, Tranter MH, Mansfield C, O'Gara P, et al. High levels of circulating epinephrine trigger apical cardiodepression in a $\beta 2$-adrenergic receptor/Gi-dependent manner: a new model of Takotsubo cardiomyopathy. Circulation 2012;126:697-706.

13. Nef HM, Möllmann H, Kostin S, Troidl C, Voss S, Weber M, et al. TakoTsubo cardiomyopathy: intraindividual structural analysis in the acute phase and after functional recovery. Eur Heart J 2007;28:2456-2464.

14. Shao Y, Redfors B, Ståhlman M, Täng MS, Miljanovic A, Möllmann H, et al. A mouse model reveals an important role for catecholamine-induced lipotoxicity in the pathogenesis of stress-induced cardiomyopathy. Eur J Heart Fail 2013;15:9-22.

15. Prasad A, Lerman A, Rihal CS. Apical ballooning syndrome (TakoTsubo or stress cardiomyopathy): a mimic of acute myocardial infarction. Am Heart J 2008;155:408-417.

16. Parodi G, Citro R, Bellandi B, Del Pace S, Rigo F, Marrani M, et al. Takotsubo cardiomyopathy and coronary artery disease: a possible association. Coron Artery Dis 2013;24:527-533.

17. Zeb M, Sambu N, Scott P, Curzen N. Takotsubo cardiomyopathy: a diagnostic challenge. Postgrad Med J 2011;87:51-59.

18. Lee PH, Song JK, Sun BJ, Choi HO, Seo JS, Na JO, et al. Outcomes of patients with stress-induced cardiomyopathy diagnosed by echocardiography in a tertiary referral hospital. J Am Soc Echocardiogr 2010;23:766771.

19. Teraoka K, Kiuchi S, Takada N, Hirano M, Yamashina A. Images in cardiovascular medicine. No delayed enhancement on contrast magnetic resonance imaging with Takotsubo cardiomyopathy. Circulation 2005;111: e261-e262.

20. Mitchell JH, Hadden TB, Wilson JM, Achari A, Muthupillai R, Flamm SD. Clinical features and usefulness of cardiac magnetic resonance imaging in assessing myocardial viability and prognosis in Takotsubo cardiomyopathy (transient left ventricular apical ballooning syndrome). Am J Cardiol 2007;100:296-301.

21. Haghi D, Fluechter S, Suselbeck T, Kaden JJ, Borggrefe M, Papavassiliu T. Cardiovascular magnetic resonance findings in typical versus atypical forms of the acute apical ballooning syndrome (Takotsubo cardiomyopathy). Int J Cardiol 2007;120:205-211.

22. Citro R, Piscione F, Parodi G, Salerno-Uriarte J, Bossone E. Role of echocardiography in takotsubo cardiomyopathy. Heart Fail Clin 2013;9:157166.

23. Crimi E, Baggish A, Leffert L, Pian-Smith MC, Januzzi JL, Jiang Y. Images in cardiovascular medicine. Acute reversible stress-induced cardiomyopathy associated with cesarean delivery under spinal anesthesia. Circulation 2008;117:3052-3053.

24. Okada T, Miyata S, Hashimoto K, Maie K, Mochizuki S. [Takotsubo cardiomyopathy associated with torsades de pointes and long QT interval: a case report]. J Cardiol 2007;50:83-88.

25. Cimarelli S, Imperiale A, Ben-Sellem D, Rischner J, Detour J, Morel O, et al. Nuclear medicine imaging of takotsubo cardiomyopathy: typical form and midventricular ballooning syndrome. J Nucl Cardiol 2008;15:137141.

26. Lancellotti P, Price S, Edvardsen T, Cosyns B, Neskovic AN, Dulgheru R, et al. The use of echocardiography in acute cardiovascular care: Recommendations of the European Association of Cardiovascular Imaging and the Acute Cardiovascular Care Association. Eur Heart J Acute Cardio- vasc Care 2015;4:3-5.

27. Meijboom WB, Meijs MF, Schuijf JD, Cramer MJ, Mollet NR, van Mieghem CA, et al. Diagnostic accuracy of 64-slice computed tomography coronary angiography: a prospective, multicenter, multivendor study. J Am Coll Cardiol 2008;52:2135-2144.

28. Marano R, De Cobelli F, Floriani I, Becker C, Herzog C, Centonze M, et al. Italian multicenter, prospective study to evaluate the negative predictive value of 16- and 64-slice MDCT imaging in patients scheduled for coronary angiography (NIMISCAD-Non Invasive Multicenter Italian Study for Coronary Artery Disease). Eur Radiol 2009;19:1114-1123.

29. Halpern EJ. Triple-rule-out CT angiography for evaluation of acute chest pain and possible acute coronary syndrome. Radiology 2009;252:332-345.

30. Eitel I, von Knobelsdorff-Brenkenhoff F, Bernhardt P, Carbone I, Muellerleile $\mathrm{K}$, Aldrovandi A, et al. Clinical characteristics and cardiovascular magnetic resonance findings in stress (takotsubo) cardiomyopathy. JAMA 2011;306:277-286.

31. Pennell DJ. Cardiovascular magnetic resonance. Circulation 2010;121: 692-705.

32. Eitel I, Behrendt F, Schindler K, Kivelitz D, Gutberlet M, Schuler G, et al. Differential diagnosis of suspected apical ballooning syndrome using contrast-enhanced magnetic resonance imaging. Eur Heart J 2008;29: 2651-2659.

33. Abdel-Aty H, Cocker M, Friedrich MG. Myocardial edema is a feature of Tako-Tsubo cardiomyopathy and is related to the severity of systolic dysfunction: insights from T2-weighted cardiovascular magnetic resonance. Int J Cardiol 2009;132:291-293.

34. Nakamori S, Matsuoka K, Onishi K, Kurita T, Ichikawa Y, Nakajima H, et al. Prevalence and signal characteristics of late gadolinium enhancement on contrast-enhanced magnetic resonance imaging in patients with takotsubo cardiomyopathy. Circ J 2012;76:914-921.

35. Rolf A, Nef HM, Möllmann H, Troidl C, Voss S, Conradi G, et al. Immunohistological basis of the late gadolinium enhancement phenomenon in tako-tsubo cardiomyopathy. Eur Heart J 2009;30:1635-1642.

36. Naruse Y, Sato A, Kasahara K, Makino K, Sano M, Takeuchi Y, et al. The clinical impact of late gadolinium enhancement in Takotsubo cardiomyopathy: serial analysis of cardiovascular magnetic resonance images. J Cardiovasc Magn Reson 2011;13:67.

37. Surapaneni P, Vittala SS, Vinales KL, Najib MQ, Chaliki HP. Atypical presentation of takotsubo cardiomyopathy. Eur J Echocardiogr 2011;12:E31.

38. Reuss CS, Lester SJ, Hurst RT, Askew JW, Nager P, Lusk J, et al. Isolated left ventricular basal ballooning phenotype of transient cardiomyopathy in young women. Am J Cardiol 2007;99:1451-1453.

39. Murthy A, Arora J, Singh A, Gedela M, Karnati P, Nappi A. Takotsubo cardiomyopathy: typical and atypical variants, a two-year retrospective cohort study. Cardiol Res 2014;5:139-144.

40. Zorzi A, Baritussio A, ElMaghawry M, Siciliano M, Migliore F, Perazzolo Marra M, et al. Differential diagnosis at admission between Takotsubo cardiomyopathy and acute apical-anterior myocardial infarction in postmenopausal women. Eur Heart J Acute Cardiovasc Care 2016;5:298-307.

41. Hoffmann U, Millea R, Enzweiler C, Ferencik M, Gulick S, Titus J, et al. Acute myocardial infarction: contrast-enhanced multi-detector row CT in a porcine model. Radiology 2004;231:697-701.

42. Lim TH, Choi SI. MRI of myocardial infarction. J Magn Reson Imaging 1999;10:686-693.

43. Abdel-Aty H, Boyé P, Zagrosek A, Wassmuth R, Kumar A, Messroghli D, et al. Diagnostic performance of cardiovascular magnetic resonance in patients with suspected acute myocarditis: comparison of different approaches. J Am Coll Cardiol 2005;45:1815-1822.

44. Schulz-Menger J. Diagnostic accuracy of CMR in biopsy-proven acute myocarditis. JACC Cardiovasc Imaging 2014;7:264-266. 